\title{
Rare Case of Adult Langerhans Cell Histiocytosis: A Case Report
}

\author{
Dinny Gustina Prihadi ${ }^{1}$, Agung Firmansyah Sumantri ${ }^{1}$, Hasyarati Agustina ${ }^{2}$, Indra \\ Wijaya $^{1}$ \\ ${ }^{1}$ Department of Internal Medicine, Faculty of Medicine, Padjadjaran University, Hasan Sadikin Hospital, Bandung, \\ Indonesia. \\ ${ }^{2}$ Department of Anatomical Pathology, Faculty of Medicine, Padjadjaran University, Hasan Sadikin Hospital, Bandung, \\ Indonesia.
}

\section{ARTICLE INFO}

Article history:

Received : 02 January 2019

Reviewed: 01 February 2019

Accepted : 12 March 2019

\section{Keywords:}

chemoterapy, Langerhans cell

histiocytis
*Corresponding author:

Dinny Gustina Prihadi

Division of Hematology and Medical

Oncology, Internal Medicine

Building 4th floor

Dr. Hasan Sadikin Hospital, Bandung

dinnyprihadi@gmail.com

\begin{abstract}
A BSTRACT
Background: Langerhans Cell Histiocytosis (LCH) is a disease characterized by proliferation and infiltration of histiocytes in various organs that is caused by local or systemic effects. LCH could affect bones, lungs, central nervous system, liver, thymus, skin, and lymph nodes. The diagnosis of LCH is difficult to enforce and rarely found in adults, namely $1-2$ cases per million per year.
\end{abstract}

Case Presentation: In Indonesia, there were very few cases of LCH in adults, one of which was found in women aged 56 years. LCH does not yet have standard treatments. The treatment is given based on location and severity, including intralesional corticosteroid injection, radiotherapy, surgical or excision curettage, and chemotherapy. Prednisone and vinblastine chemotherapy was one of chemotherapy that is given to $\mathrm{LCH}$ patients. Prognosis of $\mathrm{LCH}$ consists of various factors, one of which is the number of organs involved. This is a case report of LCH in an adult male patient with skin involvement who received chemotherapy for 6 weeks and experienced improvement.

Conclusions: LCH is not only a disease of children; it is also reported in an adult. The prognosis is related to the number of organ involved and involvement of the risk organ. LCH can recur easily and accompany malignant tumors, so follow-up and long-term observations are still needed to be done.

\section{INTRODUCTION}

Langerhans cell histiocytosis ( $\mathrm{LCH})$, also known as histiocytosis $X(H X)$, is a hyperplastic disease with unknown etiology. Histiocytosis is characterized by abnormal proliferation of histiocyte cells originating from monocytes and settling in cells in the body such as macrophages, dendritic cells, Langerhans cells, kuffer cells, microglia, and osteoclasts (1-3).

Langerhans cell histiocytosis is classified into 2 categories based on the organs involved. There are a single system and multisystem LCH. Single system LCH includes one organ, including bones (80\%), skin (33\%), lymph disorder (5-10\%), lungs (15\%), spleen (15\%), liver (15\%) and central nervous system (2-4\%). This LCH category can be unifocal or multifocal. Multisystem LCH involving two or more organs. Langerhans cell histiocytosis can occur on all age, especially in children aged 1-4 years. There are only 1-2 $\mathrm{LCH}$ cases/million in adults. Majority LCH patients are male with a sex ratio of 2: 1 , and the etiology is still unknown until now $(1,2)$.

This case involved a man diagnosed with $\mathrm{LCH}$ and underwent chemotherapy with prednisone and vinblastine for 6 weeks, who then experienced improvements. This case is interesting to be discussed because it is rare and has a good prognosis.

\section{CASE PRESENTATION}

A 40 years old man came with a lump at the inner thigh with a size of chestnut in December 2017. The lump became an open wound since March 2018. This complaint was accompanied by intermittent fever and weight loss of up to $4 \mathrm{~kg}$ in four months. The patient had decreasing appetite and night sweat. There was no joint pain, dyspnea or cough. The patient had surgery in April 
2018. In two months, a new lump grew at right inner thigh. The patient was referred to Hasan Sadikin Hospital and a second surgery was done in July 2018.

The physical examination was within normal limit. At left groin, there was an ulcer sized $10 \times 5 \times 7 \mathrm{~cm} 3$ with a muscle as the base, vascularized and hyperemic edge. The size of ulcer at right groin was $5 \times 4 \times 3 \mathrm{~cm} 3$ with subcutaneous tissue as a base and hyperemic edge.

A routine hematology test was done which showed hemoglobin $12,0 \mathrm{~g} / \mathrm{dL}$, leukocyte $11,84 \times 109 / \mathrm{mm} 3$, and thrombocyte $299 \times 109 / \mathrm{mm} 3$. The first histopathology examination showed abscess with suspicion of carcinoma. The second histopathologic examination at Hasan Sadikin Hospital showed round-oval cells morphology with hyperplastic growth, scattered pleomorphic nucleus, given half reniform and coffee bean description, hyperchromatic, mitotic found in eosinophilic cytoplasm with conclusion Langerhans cell histiocytosis at the region of left groin. Immunohistochemistry examination showed CD1a (+), focal CD20 (+) and Ki67>50\% (Figure 1).

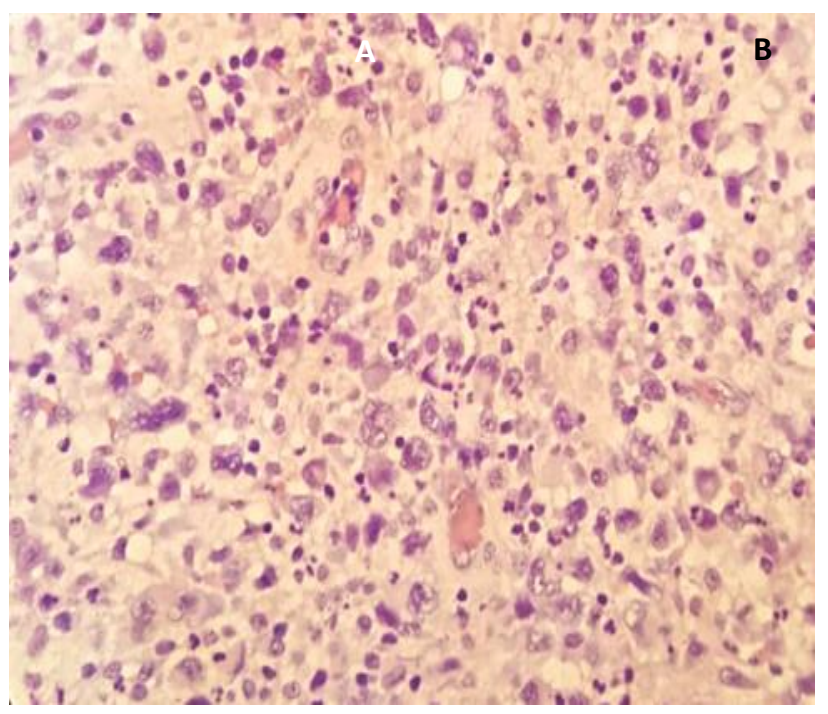

Figure 1. Histopathology of LCH with CD1a (+)

The patient was diagnosed with Langerhans cell histiocytosis. The patient received chemotherapy with prednisone $40 \mathrm{mg} / \mathrm{m} 2$ every day for 28 days with a gradual decrease in dosage every week to $30 \mathrm{mg} / \mathrm{m} 2$ and $20 \mathrm{mg} / \mathrm{m} 2$, and intravenous vinblastine $6 \mathrm{mg}$ every week for 6 weeks. While undergoing chemotherapy, patients experience mild peripheral neuropathy and neutropenia. Patients received prophylactic Granulocyte Colony Stimulating Factor (GCSF) every cycle but still experienced neutropenia.

After chemotherapy, the wound was slowly being closed (Figure 2). After 6 weeks of chemotherapy, patients still got maintenance chemotherapy with prednisone and vinblastine every 3 weeks.
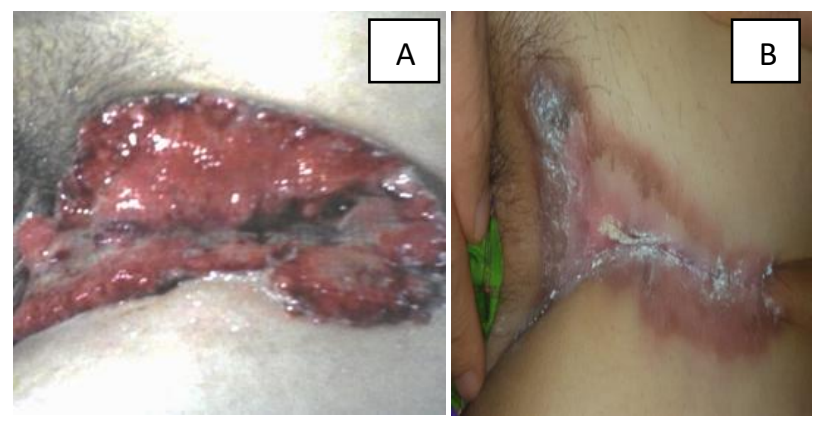

Figure 2. Lesion in the left thigh before (A) and after (B) chemoterapy

\section{DISCUSSION}

Langerhans cell histiocytosis ( $\mathrm{LCH}$ ) can involve one or many different organs, especially the bones, lungs, central nervous system, liver, thymus, skin, and lymph nodes. The level and severity of the disease vary greatly, including benign, self-limiting to death. In this case report, there was an $\mathrm{LCH}$ with skin involvement. Langerhans cell histiocytosis with skin involvement is rare, it is about $5 \%$ of the $\mathrm{LCH}$ population. It can occur at any age, most commonly in newborns and infants (2).

Diagnosis of LCH depends on clinical manifestations, physical, histopathology and electron microscopy examination. Clinical manifestations, that are often encountered, are the pain in the area of the head bone and red lesions on the skin. The accompanying symptoms are the loss of appetite, failure of body weight growth, weight loss and recurrent fever. The clinical manifestations of $\mathrm{LCH}$ depend on the age of onset, and the level of proliferation of Langerhans cells, tissues, and organs involved. The patients show limited invasive nodules and common seborrheic dermatitis rashes, which are mainly located on the trunk, scalp, hair, retro-auricular region, skin folds, and others. In addition, it can manifest as ulcers and granulomas in the groin, armpit or other areas of friction as well. In most cases, LCH patients tend to experience spontaneous improvement, but progression to multi-system $\mathrm{LCH}$ often occurs. Therefore, follow-up and reassessment of the need for treatment are needed in LCH patients. In this case, the patient experienced a boil in the groin that gets bigger and ruptured into an ulcer $(1,4)$.

Examinations include histopathology and immunohistochemistry examinations. Langerhans cell histiocytosis is characterized histologically by uncontrolled monoclonal proliferation of abnormal Langerhans cells (5). In examination with light microscopy, it will be seen that Langerhans cells accumulate in the epidermis, with multiple nuclei that looks like coffee beans and spread eosinophils. The exact diagnosis of $\mathrm{LCH}$ is done by finding Birbeck granule with an electron microscope. The distribution of Birbeck 
granule varies greatly in various tissues and affected organs.

The main examination of immunochemistry is protein S-100 and CD1a(+). Langerin (CD207) is a cell surface protein which is associated with the formation of Birbeck granules. Langerin has a higher specification than CD1a and can be tested with electron microscope method $(1,7)$. Histopathology examination shows big size round-oval cells, which are hyperplastic and spread in pleomorphic core, a part of the form is like coffee bean, hyperchromatic, and mitotic in eosinophil cytoplasm $(6,7)$. Besides the evidence of specific histopathology, this case has also shown that CD1a is positive. Overall, the case showed a clear and specific diagnosis.

The objective of LCH medication is to decrease the proliferation of histiocytes, lymphocytes, and macrophages. Langerhans cell histiocytosis management includes surgery, radiotherapy, topical corticosteroids or mechlorethamine hydrochloride solutions, thalidomide, systemic chemotherapy, and combination therapy. Nowadays, additional treatment with chemotherapy makes a better prognosis for $\mathrm{LCH}$. Optimal treatment of $\mathrm{LCH}$ remains controversial due to high clinical variability (8). Treatment depends on several factors including classification, general or focal systemic disease, organ dysfunction and age factors (1). Data for effective therapy for LCH with skin involvement are limited. Skin lesions are treated with topical steroids, surgical resection of isolated lesions, phototherapy, systemic methotrexate, 6mercaptopurine, vinblastine or vincristine, thalidomide, cladribine, and or cytarabine (9). For multi-organ diseases, especially in high-risk groups, prednisone alone or prednisone plus chemotherapy drugs, vincristine or etoposide are the most widely used according to the Histiocytosis Society protocol (10). Some chemotherapy drugs such as carboplatin, 2chlorodeoxyadenosine, chlorambucil, cyclophosphamide, cytosine arabinoside, daunomycin, etoposide, mercaptopurine, methotrexate, mechlorethamine, procarbazine, vinblastine, and vincristine showed effectivity in $50-60 \%$ of patients even though there was a possibility of relapse (11).

The front line treatment based on the association is vinblastine $6 \mathrm{mg} / \mathrm{m} 2$ i.v. weekly bolus for 6 weeks, with prednisone $40 \mathrm{mg} / \mathrm{m} 2 /$ day given orally in three divided doses for 4 weeks and then tapered over the following 2 weeks. After the first 6 weeks of treatment, disease status should be re-evaluated $(2,7)$. Evaluation of disease response is usually classified into three classifications: better, intermediate, or worse. Evaluation is classified as "better" in cases with complete resolution (non-active disease) or the presence of disease regression (better active disease), "intermediate" in cases with stable (unchanged), mixed responses with new lesions in one location, and/or regression in another site, and "worse" in cases of disease progression $(2,7)$.

The evaluation for patients with some active disease that remain in other sites is continuous treatment with vinblastine and steroids for another 6 weeks with vinblastine $6 \mathrm{mg} / \mathrm{m} 2$ i.v. weekly bolus, and prednisone $40 \mathrm{mg} / \mathrm{m} 2 /$ day orally in three divided doses for 3 days every week. Patients who have complete resolution (non-active disease) are recommended to continue with maintenance therapy for a total duration of up to 12 months with vinblastine $6 \mathrm{mg} / \mathrm{m} 2$ i.v. bolus every 3 weeks, and prednisone $40 \mathrm{mg} / \mathrm{m} 2 /$ day orally in three divided doses for 5 days every 3 weeks and $6 \mathrm{MP}$ at a dose of $50 \mathrm{mg} / \mathrm{m} 2 /$ day is added if a risk of organ involvement occurs $(2,7)$.

The prognosis of $\mathrm{LCH}$ is determined by several factors, namely, age at diagnosis, initial therapy response, number of organs involved and involvement of risk organs (hematopoietic, pulmonary, liver, spleen and bone marrow) $(7,11)$. Adult LCH patients usually had a good prognosis. The prognosis becomes poor when there is a dysfunction of lung, liver, spleen, and bone marrow, and no respond to initial treatment $(1,9)$. Patients with severe disease but respond quickly to therapy and without the involvement of the risk organs have a good prognosis with a mortality rate of $4 \%$. Whereas patients with the risk organs involvement have a mortality rate of $66 \%$. The number of organs involved also affects the prognosis: patients with 1-2 organ involvements have $0 \%$ mortality rate, patients with 3-4 organs involved have $35 \%$, patients with 5-6 organs involved have $60 \%$, and patients with $7-8$ organs involved have $100 \%$ mortality rates (9).

In our case, the patient had a good prognosis, because it occurred in adulthood with only 1 organ involved and without organ dysfunction. Management of this patient consisted of initial phase treatment of oral prednisone and intravenous vinblastine for 6 weeks in accordance with the LCH protocol $(9,11)$. Therapy according to the $\mathrm{LCH}$ protocol that uses prednisone and vinblastine can provide a survival rate of up to $100 \%$, and $65-80 \%$ free from recurrence if the patient shows a good response to the therapy (12). After undergoing treatment, the second week of skin lesions on the right thigh fold began to close, appetite increased so that the weight began to rise. At 6 weeks, the skin lesions on the left thigh fold begin to close.

\section{CONCLUSIONS}

Langerhans cell histiocytosis is not only a disease of children; it is also reported in an adult. The prognosis is related to the number of organ involved and involvement of the risk organ. LCH can recur easily and accompany malignant tumors, so follow-up and longterm observations are still needed to be done. 


\section{Acknowledgment}

We would like to thank our patients for allowing us to share his details

\section{REFERENCES}

1. Lian C, Lu Y, Shen S. Langerhans cell histiocytosis in adults: a case report and review of the literature. Oncotarget. 2016;7(4):18678-18683

2. Haupt R, Minkov M, Astigarraga I, Schafer E, Nanduri V, Jubran $R$, et al. Langerhans Cell Histiocytosis (LCH): Guidelines for Diagnosis, Clinical Work-Up, and Treatment for Patients till the Age of 18 Years. Pediatr Blood Cancer.2013;60:175-184

3. Edelweiss $M$, Medeiros L, Suster S, Moran CA. Lymph node involvement by Langerhans cell histiocytosis: a clinicopathologic and immunohistochemical study of 20 cases. Human Pathology. 2007;38:1463-1469

4. Braier J, Ciocca M, Latella A, Davila MG, Drajer M, Imventarza O. Cholestasis, sclerosing cholangitis, and liver transplantation in Langerhans cell histiocytosis. Med Pediatr Oncol 2002;38:178-182

5. Rautenbach K, Stones DK. Langerhans cell histiocytosis: A case presentation and literature review. S Afr J CH. 2013;7(2):65-67
6. Kim SH, Choi MY. Langerhans Cell Histiocytosis of the Rib in an Adult: A Case Report. Case Rep Oncol. 2016;9(1):83-88

7. Minkov M, Grois N, McClain K, Nanduri V, Galindo CR, Klupp IS, et al. Langerhans Cell Histiocytosis. Histiocyte Society Evaluation and Treatment Guidelines. 2009.

8. Kumar YP, Agrawal J, Mohanlakshmi J, Kumar PS. Langerhans cell histiocytosis revisited: Case report with review. Contemp Clin Dent. 2015;6(3):432436.

9. Allen CE, Ladisch S, McClain KL. How I treat Langerhans cell histiocytosis. Blood. 2015 Jul;126(1):26-35

10. Jeunon KT, Sousa MAJ, Rodrigues NS, Lopes R. Langerhans cell histiocytosis - a case report. Dermatol Pract Concept. 2012;2(1):4

11. Weitzman S, Egeler RM. Langerhans cell histiocytosis: update for the pediatrician. Current Opinion in Pediatrics. 2008;20:23-29

12. Totadri S, Bansal D, Trehan A, Srinivasan R, Varma $\mathrm{N}$, Kakkar N, et al. The 5-Year EFS of Multisystem LCH With Risk-Organ Involvement Is Suboptimal: A Single-center Experience From India. J Pediatr Hematol Oncol. 2016;38(1):e1-e5. 